\title{
Bloqueio "3 em 1" por Via Anterior: Bloqueio Parcial, Completo ou Superdimensionado? Correlação entre Anatomia, Clínica e Radio Imagens *
}

\author{
Anterior "3-in-1" Blockade: Partial, Total or Overdimensioned Block? \\ Correlation between Anatomy, Clinic and Radio Images \\ Karl Otto Geier ${ }^{1}$
}

\begin{abstract}
RESUMO
Geier KO - Bloqueio “3 em 1" por Via Anterior: Bloqueio Parcial, Completo ou Superdimensionado? Correlação entre Anatomia, Clínica e Rádio Imagens
\end{abstract}

JUSTIFICATIVA E OBJETIVOS: O clássico bloqueio "3 em 1" por
via anterior tem suscitado divergências quanto ao envolvimento
anestésico de seus três nervos participantes, o femoral, o cutâneo
lateral da coxa o o obturador. O objetivo deste estudo é verificar o
desfecho do bloqueio "3 em 1" por via anterior, através das
técnicas: injeção única (G1), cateteres curtos (G2) e cateteres
longos (G3). Os bloqueios "3 em 1", clinicamente identificados
como completos ou superdimensionados foram, adicionalmente,
investigados por meio de rádio imagem. MÉTODO: A identificação do espaço subfascial ilíaco nos bloqueios "3 em 1" com injeção única ou com cateteres foi feita pela perda de resistência ao ar. Em vários eventos dolorosos, o volume anestésico administrado variou entre 30 e $40 \mathrm{ml}$ e a introdução cranial dos cateteres foi até $18 \mathrm{~cm}$ no espaço subfascial ilíaco. Quando a pesquisa clínica apontava envolvimento do nervo obturador ou de outro nervo adicional ao bloqueio "3 em 1", complementava-se a investigação com estudo radiográfico e tomodensiométrico com o propósito de estabelecer correspondência com a anatomia pélvica.

RESULTADOS: O envolvimento dos nervos fêmoro cutâneo lateral e obturador não foram constantes, ao contrário do nervo femoral. No estudo, nenhum bloqueio "3 em 1" completo com injeção única (G1) se manifestou, e sim um bloqueio "2 em 1", com a participação eventual do ramo femoral do nervo genitofemoral (bloqueio " 2,5 em 1"). Contudo, quando foram utilizados cateteres curtos (G2), obteve-se bloqueio "3 em 1" em apenas um paciente, ao passo que com cateteres longos (G3) introduzidos no sentido cefálico até 18 $\mathrm{cm}$ no espaço subfascial ilíaco, três bloqueios "3 em 1" superdimensionados foram registrados, pelo envolvimento adicional dos nervos fibular comum em dois pacientes e o nervo tibial em um paciente.

CONCLUSÕES: Apesar da pequena amostra, com injeção única (G1), sempre se obteve um bloqueio " $2 \mathrm{em} 1$ " ou "2,5 em 1", sem a participação do nervo obturador. Com cateter curto (G2), o

\footnotetext{
* Recebido do (Received from) Hospital Municipal de Pronto Socorro de Porto Alegre, RS

1. Anestesiologista do Hospital Municipal de Pronto Socorro de Porto Alegre/RS; Anestesiologista Colaborador da Clindor do Hospital São Lucas da PUC/RS; Mestre em Cirurgia pela UFRGS
}

Apresentado (Submitted) em 21 de julho de 2003

Aceito (Accepted) para publicação em 06 de novembro de 2003

Endereço para correspondência (Correspondence to)

Dr. Karl Otto Geier

Rua Cel. Camisão, 172

90540-030 Porto Alegre, RS

E-mail:karlotto@terra.com.br

(C) Sociedade Brasileira de Anestesiologia, 2004 bloqueio "3 em 1" foi classificado como completo em 6,6\% dos casos (um paciente). Porém, com cateter longo (G3), o resultado tende a ser mais previsivel em relação aos outros grupos, especialmente quando o cateter alcança o espaço paravertebral lombossacral, resultando num bloqueio "3 em 1" completo em $20 \%$ dos casos (três pacientes) ou, raramente, num bloqueio "3 em 1" superdimensionado em 13,2\% dos casos (dois pacientes).

Unitermos: TÉCNICAS ANESTÉSICAS, Regional: bloqueio "3 em 1"

\section{SUMMARY}

Geier KO - Anterior "3-in-1" Blockade: Partial, Total or Overdimensioned Block? Correlation between Anatomy, Clinic and Radio Images

BACKGROUND AND OBJECTIVES: Classic anterior 3-in-1 blockade has been questioned as to the anesthetic involvement of its three participant nerves: femoral, lateral cutaneous of thigh and obturator. This study aimed at evaluating the outcome of anterior 3-in-1 blockade through: single injection (G1), short catheters (G2) and long catheters (G3). 3-in-1 blockades identified as total or overdimensioned were additionally investigated by radio images. METHODS: The identification of iliac subfascial space in 3-in-1 blockades with single injection or catheters has been made by loss of resistance to air. In several painful events, anesthetic volume has varied 30 to $40 \mathrm{~mL}$ and cranial catheters introduction was up to $18 \mathrm{~cm}$ in the iliac subfascial space. When clinical research would point to the involvement of the obturator nerve or other nerve additional to 3-in-1 blockade, investigation was complemented by radiographic or TC-Scan studies aiming at establishing correspondence with pelvic anatomy.

RESULTS: The involvement of lateral cutaneous of thigh and obturator nerves has not been constant, as opposed to the femoral nerve. No total 3-in-1 blockade with single injection (G1) was observed in our study, but rather 2-in-1, with eventual participation of the femoral branch of the genitofemoral nerve (2.5-in-1 blockade). However, when short catheters were used (G2), there has been 3-in-1 blockade in just one patient, while with long catheters (G3) introduced in the cephalad direction until $18 \mathrm{~cm}$ in the iliac subfascial space, three overdimensioned 3-in-1 blockades were recorded, with the additional involvement of common fibular nerve in two patients and tibial nerve in one patient.

CONCLUSIONS: In spite of the small sample size, single injection (G1) has always provided 2-in-1 or 2.5-in-1 blockade without the participation of the obturator nerve. With short catheters (G2) 3-in-1 blockade was classified as total in $6.6 \%$ of cases (one patient). With long catheters (G3), however, results seem to be more predictable as compared to the other groups, especially when the catheter reaches the lumbosacral paravertebral space, resulting in total 3-in-1 blockade in $20 \%$ of cases (3 patients) or more uncommonly, in overdimensioned 3-in-1 blockade in $13.2 \%$ of cases (2 patients).

Key Words: ANESTHETIC TECHNIQUES, Regional: 3-in-1 block 


\section{INTRODUÇÃO}

$\mathrm{O}$ bloqueio "3 em 1" por via anterior, originalmente apresentado por Winnie e col. em $1973^{1}$, teve como finalidade anestesiar simultaneamente, com apenas uma injeção, os nervos femoral, o cutâneo lateral da coxa e o obturador, componentes do plexo lombar, acessando o espaço paravascular femoral logo abaixo do ligamento inguinal. A técnica compreendia o desencadeamento de parestesias com agulha sobre o nervo crural seguido por injeção de 20 ou mais mililitros de uma solução anestésica. Recomendavam pressionar os tecidos imediatamente distais à agulha com o propósito de favorecer a dispersão da solução anestésica no espaço subfascial ilíaco durante a injeção. Inicialmente, houve êxito nos resultados ${ }^{1}$, com bloqueio " 3 em 1" completo. Porém, à medida que a técnica foi se tornando popular, constatou-se na prática clínica, que o acometimento dos três nervos, após injeção única nem sempre ocorria. Várias publicações mostraram que o nervo obturador ${ }^{2}$ com maior freqüência que o nervo cutâneo lateral da coxa ${ }^{3}$ falhava, ao contrário do que acontecia com o nervo femoral ${ }^{4}$, caracterizando um bloqueio " $3 \mathrm{em}$ 1" parcial ou um bloqueio "2 em 1". Todavia, raríssimos bloqueios " 3 em 1" ipsilaterais e superdimensionados foram documentados ${ }^{5,6}$. Orientado pelas referências anatômicas superficiais sugeridas por Vloka e col. ${ }^{7}$, observaram-se durante 45 bloqueios " 3 em 1" consecutivos, com injeção única ou com cateteres, o envolvimento parcial e ipsilateral do plexo lombar. Os desfechos clínicos, que identificaram um bloqueio "3 em 1" completo ou superdimensionado face ao envolvimento adicional de nervos lombossacros, foram correlacionados à anatomia e à um estudo de imagens radiológicas correspondentes.

\section{MÉTODO}

Após aprovação pela Comissão de Ética Médica do Hospital e pelos pacientes, foram realizados 45 bloqueios " 3 em 1", pela abordagem paravascular femoral em três grupos de quinze pacientes, utilizando seringas de vidro ou de plástico, apropriadas para o teste de perda de resistência ao ar, aguIhas descartáveis com ponta cortante, cateteres curtos com orifício terminal e cateteres longos com três orifícios laterais:

Grupo 1 - Constou de 15 bloqueios "3 em 1", realizados com injeção única mediante agulha descartável $35 \times 7$ ou $40 \times$ 7 e acoplada a uma seringa contendo ar e com mínimo atrito aos movimentos do êmbolo. O conjunto agulha-seringa foi introduzido $30^{\circ}$ a $45^{\circ}$ em relação à pele até o espaço subfascial ilíaco, utilizando-se lidocaína a 1,5\% com adrenalina a 1:300.000 (30 a $40 \mathrm{ml})$.

Grupo 2 - Abrangeu 15 pacientes que se submeteram ao bloqueio " 3 em 1" com cateteres venosos curtos 18G, sendo seis Introcan ${ }^{\circledR}$ da B. Braun de $45 \mathrm{~mm}$ de comprimento e nove da Nipro Medical Ltda. de $50 \mathrm{~mm}$ de com- primento, ambos com ponta Quincke, introduzidos num ângulo agudo similar ao grupo 1. No instante da identificação do espaço subfascial ilíaco, equivalente à segunda perda de resistência, metade do volume anestésico era injetado, a agulha do cateter venoso imobilizada e o cateter introduzido, no sentido cranial, em toda sua extensão, sob movimentos giratórios no sentido horário e anti-horário ao longo do eixo da agulha seguido pela retirada da agulha do cateter venoso. O volume de anestésico inicial facilitava a passagem dos cateteres, utilizando-se bupivacaína a 0,25\% com adrenalina a 1:400.000 (30 a $40 \mathrm{ml})$.

Grupo 3 - Os bloqueios "3 em 1" foram realizados com cateteres peridurais longos $18 \mathrm{G}$, nove Perifix ${ }^{\circledR}$ da B. Braun e seis Minipack ${ }^{\circledR}$ da Portex. Todos foram introduzidos no sentido cranial entre 15 e $18 \mathrm{~cm}$, no espaço subfascial ilíaco. Os cateteres Perifix ${ }^{\circledR}$ através de seu cateter guia sem o auxílio do estimulador de nervo periférico e os cateteres Minipack ${ }^{\circledR}$ através da cânula de um cateter venoso $18 \mathrm{G}$, todos previamente introduzidos conforme descrito no grupo 2. Em todos os cateteres se acoplou um filtro antibacteriano, o anestésico utilizado foi bupivacaína a 0,25\% com adrenalina 1:400.000 (40 ml).

A avaliação clínica sensitiva e motora do bloqueio " 3 em 1" dos grupos, foi feita pela pesquisa da analgesia dos dermátomos (inervação cutânea) e/ou dos miótomos (inervação muscular) em queimaduras e ferimentos lácero-corto-contusos, todos com indicação cirúrgica, e dos esclerótomos (inervação óssea) em pacientes com traumatismos de coxa acompanhados por fratura do terço médio do fêmur e joelho. Nos casos em que a investigação sensorial cutânea, muscular e óssea não foi possível por causa das bandagens ou imobilizações, o próprio alívio da dor foi o referencial efetivo do bloqueio "3 em 1". Para as cirurgias eletivas sob analgesia através dos cateteres com extensão ao período pós-operatório, a aferição da sensibilidade dos dermátomos obedeceu a seqüência: dor (tração dos folículos pilosos/picada de aguIha), temperatura (gelo) e tato, seguida pela motricidade dos miótomos, conforme já descrito ${ }^{8}$, desde que compatíveis com a lesão. Todas as investigações clínicas realizadas foram comparadas com o lado homólogo.

O estudo anatômico foi acoplado ao estudo radiológico, este último realizado nos bloqueios com os cateteres, curtos ou longos (peridural e Contiplex ${ }^{\circledR}$ ), que resultaram no envolvimento clínico dos clássicos três nervos e de outro nervo adicional. Inicialmente, um a dois mililitros de contraste, identificavam o cateter no espaço subfascial ilíaco, e depois um volume maior de contraste (Omnipaque ${ }^{\circledR} 300$ $\mathrm{mg} / \mathrm{ml}$ ) diluído com anestésico local registrava a dispersão nas possíveis três zonas radiológicas lombopélvicas: zona $A$ (pélvica medial), zona B (pélvica lateral) e zona $C$ (paravertebral) ${ }^{5}$ (Figura 1 ). Se a exploração radiológica contrastada sobrepunha ao percurso anatômico dos nervos interessados, prosseguia-se com um estudo tomográfico computadorizado. 


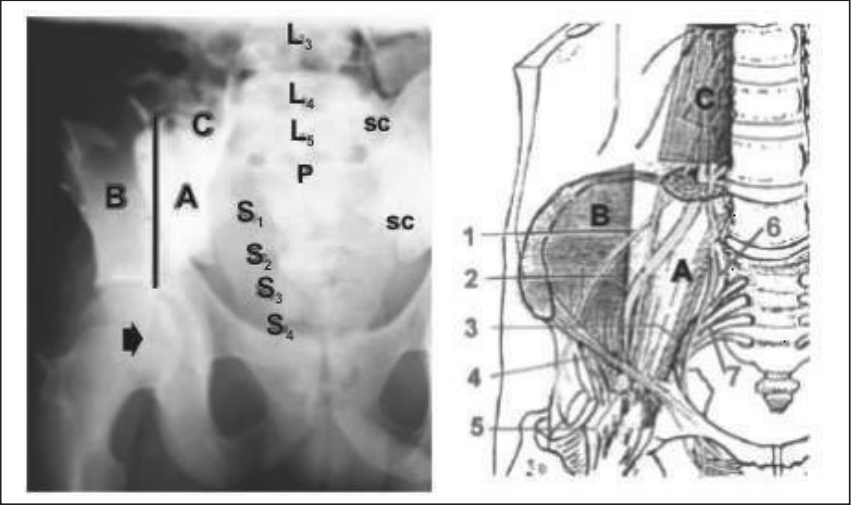

Figura 1 - Representação das Zonas Radiológicas ${ }^{5}$ A, B, C onde Transitam os Componentes do "Bloqueio 3:1"por Via Femoral Esquerda: A impregnação de contraste numa incidência ântero-posterior pode revelar o envolvimento individual ou participativo dos clássicos três nervos do "bloqueio $3: 1$ ". Seta $=$ cateter; P = promontório sacral; SC = articulação sacro ilíaca $S_{1} ; S_{2} ; S_{3} ; S_{4}=$ forâmens sacrais; $L_{3}, L_{4}, L_{5}=$ corpos vertebrais Direita:; 1 = nervo cutâneo lateral da coxa; 2 = nervo femoral; 3 $=$ nervo obturador; $4=$ nervo genitofemoral; $5=$ local de punção; 6 = tronco lombossacro; 7 = plexo sacral

\section{Análise Estatística}

Os dados demográficos foram expressos em Média \pm DP. Para as variáveis categóricas dos três grupos sobre o acometimento individual dos nervos do bloqueio " 3 em 1", os resultados foram em freqüências relativas, seguido pelo teste $x^{2}$ para determinar significância dos resultados, com o valor de $p<0,05$ considerado estatisticamente significativo.

\section{RESULTADOS}

Os dados demográficos e os procedimentos nos três grupos estão representados nas tabelas I e II.

Tabela I - Dados Demográficos dos Grupos (Média \pm DP)

\begin{tabular}{lccc}
\hline Grupos & Idade & Altura & Peso \\
\hline G1- Injeção Única & $27,50 \pm 7,01$ & $169,33 \pm 8,19$ & $67,58 \pm 5,42$ \\
G2 - Cateter curto & $27,17 \pm 6,13$ & $166,58 \pm 6,01$ & $65,42 \pm 4,95$ \\
G3 - Cateter longo & $23,67 \pm 4,70$ & $171,92 \pm 9,88$ & $70,92 \pm 8,48$ \\
\hline
\end{tabular}

Tabela II - Procedimentos Realizados nos Três Grupos

\begin{tabular}{lccc}
\hline Indicações & $\begin{array}{c}\text { G1=Injeção } \\
\text { Única \#* }\end{array}$ & $\begin{array}{c}\text { G2=Cateter } \\
\text { Curto }\end{array}$ & $\begin{array}{c}\text { G3=Cateter } \\
\text { Longo }\end{array}$ \\
\hline Área doadora de pele *。 & 13 & 10 & \\
$\begin{array}{l}\text { Troca de curativos }{ }^{* \circ} \\
\begin{array}{l}\text { Desbridamentos } \\
\text { seriados * }\end{array}\end{array}$ & 03 & 06 \\
$\begin{array}{l}\text { Fratura terço médio de } \\
\text { fêmur \# }\end{array}$ & & 05 \\
Fratura do quadril * & & & 01 \\
Fratura do joelho * & & & 01
\end{tabular}

Nevralgia do nervo safeno

01 interno •

Extração da veia safena magna ${ }^{\circ}$

Mialgia dos adutores da coxa

Dor por picada de aracnídeo (aranha)

$$
n=15 \quad n=15 \quad n=15
$$

Todos os cateteres foram 18G; agulha descartável 35 × 7 ;

* (analgesia pós-operatória); ${ }^{\circ}$ (cirurgia/procedimentos dolorosos);

\# (dor pré-operatória); • (dor crônica)

O envolvimento do dermátomo femoral (nervo misto) foi freqüente, com fraqueza muscular do quadríceps femoral e quase com a mesma freqüência, perda de sensibilidade no dermátomo cutâneo lateral da coxa (nervo sensitivo). Em alguns casos, foi observada perda de sensibilidade na face medial de quase toda a coxa onde os dermátomos femoral e obturador se sobrepõem, com limites imprecisos, porém, nem sempre acompanhado por paresia muscular adutora. Dependendo da morbidade do membro inferior, a motricidade ativa (flexão da coxa - nervo femoral e adução da coxa nervo obturador) e passiva (manter joelho flexionado com a região plantar apoiada sobre o leito, sem pender o membro lateralmente - nervo obturador) também foram avaliadas conforme previamente descrito ${ }^{8}$. Analgesia e anestesia de dermátomos adicionais aos três clássicos nervos do bloqueio " 3 em 1", foram constatadas nos territórios do ramo femoral do nervo genitofemoral em sete pacientes $(46,6 \%)$ e, no território do nervo fibular comum em dois pacientes $(13,2 \%)$, caracterizando um bloqueio superdimensionado " $3,5 \mathrm{em} 1$ " sendo que em um destes observou-se adicionalmente, comprometimento parcial do nervo tibial $(6,6 \%)$, traduzindo-se praticamente, em um bloqueio "4 em 1" (Tabela III).

Tabela III - Envolvimento dos Nervos Periféricos do Bloqueio Anterior "3 em 1" ( $n=45)$

\begin{tabular}{|c|c|c|c|c|c|c|}
\hline & \multicolumn{3}{|c|}{ Bloqueio "3 em 1" } & \multicolumn{3}{|c|}{ Nervos Adicionais ao Bloqueio "3 em 1" } \\
\hline & $\begin{array}{c}\text { Nervo Femoral } \\
(\mathrm{S} / \mathrm{M})\end{array}$ & $\begin{array}{c}\text { Nervo Cutâneo } \\
\text { Lateral da Coxa (S) }\end{array}$ & $\begin{array}{c}\text { Nervo Obturador } \\
(\mathrm{S} / \mathrm{M})\end{array}$ & $\begin{array}{c}\text { Nervo } \\
\text { Genitofemoral (S) }\end{array}$ & $\begin{array}{l}\text { Nervo Fibular } \\
\text { Comum (S/M) }\end{array}$ & $\begin{array}{l}\text { Nervo Tibial } \\
(\mathrm{S} / \mathrm{M})\end{array}$ \\
\hline G1 - Injeção única $(n=15)$ & $15 \backslash 15(100 \%)$ & $13 \backslash 15(86,6 \%)$ & $0 \backslash 15$ & $4 \backslash 15(26,4 \%)$ & $0 \backslash 15$ & $0 \backslash 15$ \\
\hline G2 Cateter curto $(n=15)$ & $15 \backslash 15(100 \%)$ & $14 \backslash 15(93,2 \%)$ & $1 \backslash 15(6,6 \%)$ & $7 \backslash 15(46,6 \%)$ & $0 \backslash 15$ & $0 \backslash 15$ \\
\hline G3 Cateter longo $(n=15)$ & $15 \backslash 15(100 \%)$ & $13 \backslash 15(86,6 \%)$ & $2 \backslash 15(13,2 \%)$ & $7 \backslash 15(46,6 \%)$ & $2 \backslash 15(13,2 \%)$ & $\begin{array}{c}1 \backslash 15(6,6 \%) \\
\text { medial plantar }\end{array}$ \\
\hline
\end{tabular}

(S) Sensibilidade; (M) Motricidade; Acometimento entre os nervos dos grupos: NS (teste $X^{2}$ ) 
Não obstante os 15 bloqueios ditos "3 em 1" com injeção única não resultarem no envolvimento motor do nervo obturador, esses bloqueios foram classificados como sendo " 2 em 1 " e, portanto, nenhum estudo radiológico foi realizado coma repetição do mesmo volume e massa de anestésico local. Todavia, nos 30 pacientes com cateteres curtos (quinze) e longos (quinze) instalados no espaço subfascial dos músculos pélvico-lombares, que manifestaram clinicamente perda de sensibilidade em dermátomo(s) ectópico(s) ao bloqueio "3 em 1", foram inicialmente investigados através de radiografias ântero-posterior com um a dois mililitros de contraste para identificar o posicionamento do cateter (Figura 2).

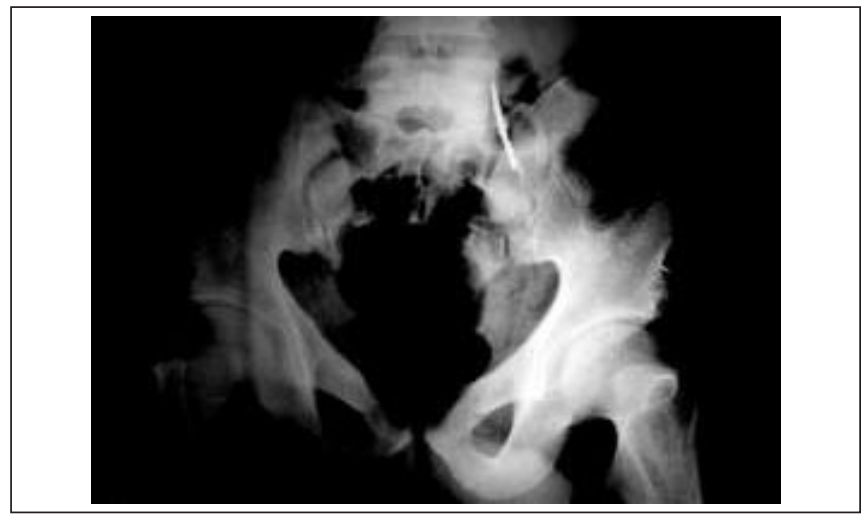

Figura 2 - Um a Dois mililitros de Contraste pelo Cateter Contiplex ${ }^{\circledR}$ revela sua Posição nas Zonas Radiológicas Esquerdas A e C num Paciente com Comprometimento Doloroso no Território do Nervo Obturador. A Articulação Sacro llíaca é Visível, sendo Considerada um Referencial Radiológico do Trajeto do Nervo Obturador

Dentre os pacientes com cateteres curtos, apenas um desenvolveu bloqueio "3 em 1" completo (verdadeiro) com alteração de sensibilidade de um dermátomo extra aos três clássicos nervos do bloqueio "3 em 1". As imagens radiográficas contrastadas identificaram as zonas radiológicas pélvicas $A$ (espaço subfascial do músculo psoas) e $B$ (espaço subfascial do músculo ilíaco) porém, sem ascensão medial pela zona A. Em nenhum momento o contraste atingiu a zona $\mathrm{C}$ (região paravertebral). Por outrolado, por serem os cateteres longos predestinados à uma introdução mais cranial entre 15 e $18 \mathrm{~cm}$ no espaço subfascial ilíaco, não exibiram, na sua totalidade, manifestações clínicas que sugerissem ascensão cranial até a zona radiológica C com anestesia do nervo obturador. A tendência para enrolamentos e dobras durante a introdução destes últimos cateteres, frusta seu aporte à região paravertebral lombar, região onde se encontram confinados os três nervos, o que explica a ausência de bloqueio do nervo obturador.

Dos quinze pacientes com cateter longo, dois apresentaram o posicionamento do cateter na altura de $L_{5}-L_{4}$ (Figura 3 ) numa freqüência de $13,2 \%$. As tomografias computadorizadas desses pacientes mostraram o contraste situado nas zonas radiológicas A, B, C (Figuras 4 e 5), inclusive com envolvimento de dermátomos adicionais aos do bloqueio " 3 em 1 ". Este acontecimento ocorreu num paciente com avulsão traumática de pele do dorso do pé e submetido à enxertia da re-

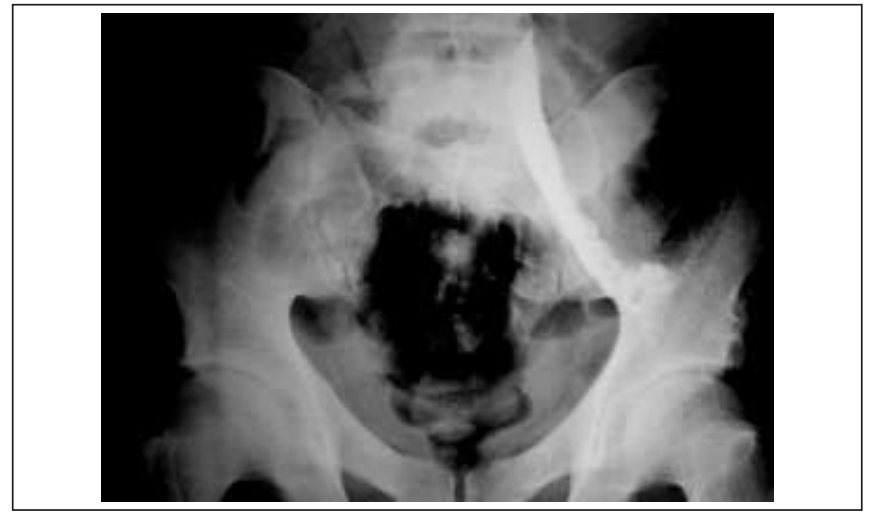

Figura 3 - O mesmo Paciente da figura 2 Identificando o Anestésico Local Misturado ao Contraste, com Dispersão ao Longo do $5^{\circ}$ Fascículo Muscular do Psoas, às Zonas Radiológicas A e $\mathrm{C}^{5} \mathrm{O}$ que correspondeu aos achados clínicos de um bloqueio "3,5:1" com envolvimento dos nervos cutâneo lateral da coxa e femoral (ambos na zona radiológica B), obturador (zona radiológica $\mathrm{Ae} \mathrm{C}$ ) e do ramo femoral do nervo genitofemoral (zonas radiológicas transicionais $A$ e $B$ ) e do tronco lombossacro $\left(\mathrm{L}_{4}-\mathrm{L}_{5}\right)$

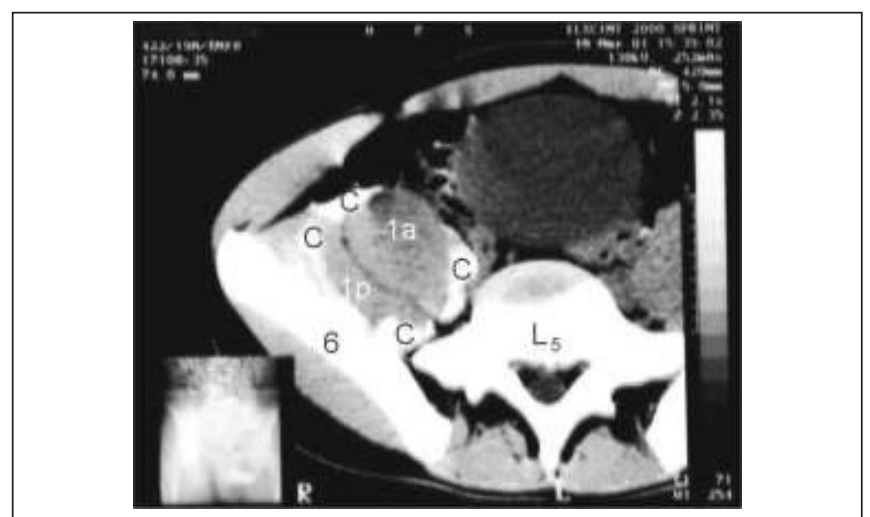

Figura 4 - Imagem Tomodensiométrica em $\mathrm{L}_{5}$ de Um dos Dois Pacientes que Exibiram Bloqueio "3 em 1" Superdimensionado envolvendo o Tronco Lombossacro resultando na Anestesia do Nervo Fibular Comum e, parcialmente, o Nervo Tibial Observa-se o contraste (C) envolvendo e difundindo-se no músculo ilíaco e nas duas porções do músculo psoas; 1 a = porção maior ou anterior do músculo psoas; $1 \mathrm{~b}=$ porção menor ou posterior do músculo psoas; $6=$ osso ilíaco

gião, cujo bloqueio femoral "3 em 1" destinava-se à analgesia intermitente pós-operatória da área doadora ântero-lateral da coxa. Para surpresa, além de efetiva analgesia da área doadora, o paciente referiu perda de sensibilidade tátil/dolorosa de toda face lateral da perna e do dorso do pé, correspondente ao dermátomo do nervo fibular comum $\left(L_{4}-L_{5}-S_{1}-S_{2}\right)$ acompanhado por sensação de aumento de volume e de temperatura do pé, característica de um bloqueio simpático. Esse mesmo paciente não conseguia cruzar o membro inferior alvo sobre seu homólogo, devido ao intenso bloqueio motor dos músculos adutores (nervo obturador), embora esboçasse leve flexão do pé e dos dedos (nervo tibial posterior) em contraste com o outro pé. Neste paciente confirmou-se a perda de sensibilidade na face medial plantar, correspondente ao dermátomo do nervo tibial. As imagens 


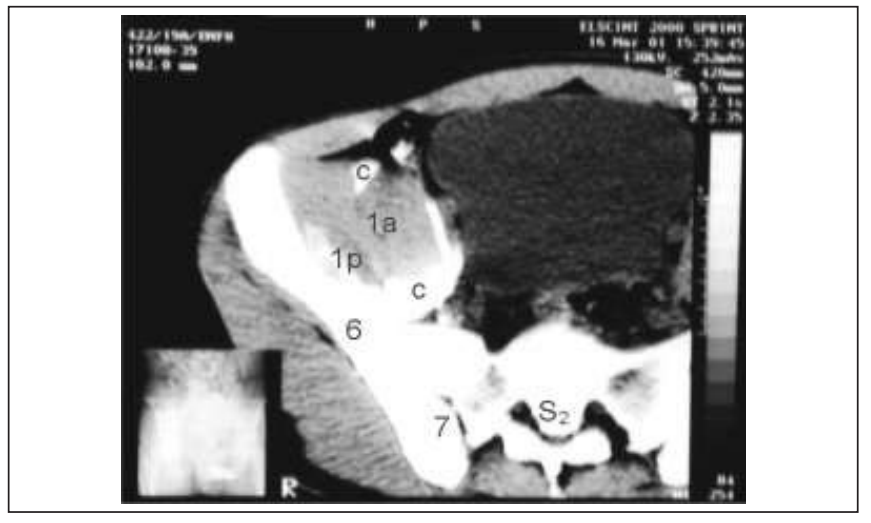

Figura 5 - Imagem Tomodensiométrica em $\mathrm{S}_{2}$ do mesmo Paciente da figura 4 cujo Contraste (C) atingiu o Tronco Lombossacro, resultando em Anestesia dos Nervos Fibular Comum e Tibial $\mathrm{C}=$ contraste difundindo-se no músculo ilíaco e nas duas porções do músculo psoas, na altura da articulação sacro ilíaca, parcialmente visível (7); 1a = porção maior ou anterior do músculo psoas; $1 b=$ porção menor ou posterior do músculo psoas; 6 = osso ilíaco

tomográficas (Figuras 4 e 5) mostraram difusão às zonas $\mathrm{Ce}$ A sacral $\left(\mathrm{S}_{2}\right)$ (Figura 5$)$.

\section{DISCUSSÃO}

Anatomicamente, a inervação do membro inferior é feita pelo plexo lombar $\left[\left(T_{12}\right)-L_{1}-L_{5}\right]$ e plexo sacral $\left(L_{4}-S_{3}\right)^{9,10}$. A união desses dois plexos é mediada pelo tronco lombossacral $\left(L_{4}-L_{5}\right)$. Os dermátomos do femoral $\left(L_{2}-L_{4}\right)$ e do obturador $\left(L_{2}-L_{4}\right)$, este último predominantemente motor (através das raízes $L_{3}$ e $L_{4}$ ), se sobrepõem na face interna da coxa sem limites precisos ${ }^{11}$ ou com distribuição mais proximal ${ }^{12}$ que distal ou mesmo ausente ${ }^{13}$. Conforme Bouaziz e col. ${ }^{14}$ o dermátomo do obturador encontra-se ausente em quase $60 \%$ dos casos. A questão adquire mais importância numa das edições do livro de Gray ${ }^{9}$ onde o autor não faz alusão ao dermátomo do obturador. A confusão entre os dermátomos do obturador e do femoral continua persistindo ${ }^{15}$. Os nervos femoral e cutâneo lateral da coxa estão situados mais lateralmente entre os músculos ilíaco e psoas, e a fascia ilíaca que os reveste ${ }^{16}$. Enquanto a fascia ilíaca no sentido distal delimita o espaço paravascular femoral na região inguinal, o nervo obturador, ao se dirigir desde sua origem ao membro inferior, o faz mais posteriormente, por fora desse espaço e medialmente ao músculo psoas maior ${ }^{17}$, tornando sua abordagem difícil pela técnica de injeção única, diferentemente do que ocorre quando a solução anestésica é liberada pela ponta de um cateter posicionado na zona radiológica A ou ainda, porvia posterior onde o anestésico é depositado diretamente na zona radiológica $\mathrm{C}$. O nervo obturador segue seu trajeto caudal, nem sempre acompanhando os nervos femoral e cutâneo lateral da coxa. No nível $L_{4}-L_{5}$, enquanto o primeiro cursa, com freqüência variável, por fora ou perifericamente entre as duas partes do músculo psoas maior, ambas revestidas por uma tênue fáscia ${ }^{17,18}$, os dois últimos guardam uma relação mais constante entre si dentro da massa muscular ${ }^{19}$, distanciando-se um do outro, na altura do promontório sacral
$\left(L_{5}-S_{1}\right)$. O nervo obturador cursa para a pequena pelve e os outros dois para a grande pelve. Na altura do forâmen que recebe seu nome, o nervo obturador divide-se em dois ramos: ramo anterior ou superficial e ramo posterior ou profundo. O ramo anteriorou superficial se dirige à articulação do quadril, e à superfície cutânea medial da perna que, anastomosando-se ao nervo safeno interno e aos múltiplos ramos cutâneos mediais do nervo femoral, formam o plexo subsartorial, cuja projeção sensitiva cutânea gera confusões quanto aos testes de sensibilidade de seus dermátomos correspondentes. No terço medial da coxa, o ramo anterior ou superficial, predominantemente sensitivo, se dirige aos tecidos superficiais, tendo pouca participação motora nos músculos adutor longo, gracilis e adutor curto ${ }^{11}$. O ramo profundo ou posterior, predominantemente motor, se dirige aos músculos obturador externo, adutor magno e adutor curto, anastomosando-se em seguida ao ramo superficial, projetando-se à face posterior da região poplítea e à membrana sinovial do joelho. Agrande maioria das observações clínicas do bloqueio " 3 em 1 " verifica apenas o comprometimento sensitivo e não a pesquisa motora do nervo obturador, causando interpretações equivocadas sobre sua extensão ${ }^{20}$. Critérios objetivos e bem definidos ${ }^{21}$ devem, pois, reger a pesquisa clínica de todo e qualquer bloqueio anestésico aos propósitos destinados, verificando a abolição sensorial e motora, respectivamente, dos tecidos superficiais (cutâneos) e profundos (músculos, articulações, vísceras e vasos).

Evidências técnicas no resultado do bloqueio "3 em 1" confirmam que, para a identificação do espaço paravascular femoral não se impõe, necessariamente, a técnica do estimulador de nervo periférico ${ }^{21}$ ou de parestesias ${ }^{1}$ através do contato da agulha com o nervo. A perda de resistência é um método adequado, adaptando-se na identificação de espaços virtuais ${ }^{21-23}$ entre tecidos de densidades diferentes em vários bloqueios periféricos ${ }^{24}$.

O recurso das diversas modalidades de imagens radiológicas contrastadas em anestesia regional começa a ter importância, repercutindo intensamente no aprendizado das técnicas anestésicas regionais ${ }^{16}$, no diagnóstico e tratamento das doenças dolorosas ${ }^{25}$. Apesar das imagens unidimensionais, as evidências radiológicas contrastadas ântero-posteriores das três zonas radiográficas ${ }^{5}$ devem ser interpretadas com base na dispersão do contraste no compartimento subfascial dos músculos ilíaco e psoas maior, por onde transitam os componentes nervosos do bloqueio femoral " 3 em 1 ". O nervo obturador percorre, distalmente, a zona A (região pélvica medial), o nervo femoral, transita distalmente, entre a zona A e o limite interno da zona B (região pélvica lateral), e o nervo lateral cutâneo da coxa dirige-se distalmente à zona $B$. Azona $\mathrm{C}$ (região paravertebral lombar) corresponde à metade proximal do músculo psoas ${ }^{5}$ (Figura 1 ) onde, uma única injeção por via posterior é mais factível anestesiar todos os três integrantes do bloqueio " 3 em 1" ${ }^{26}$, quando se compara com a abordagem anterior. Ao se estabelecer um bloqueio "3 em 1" com cateteres longos, as imagens contrastadas são em geral alongadas, fusiformes e bem delimitadas, terminando, quase sempre, lateralmente aos corpos vertebrais Vol. 54, No 4, Julho - Agosto, 2004 
$\mathrm{L}_{3}-\mathrm{L}_{4}$ no nível dos discos intervertebrais, correspondendo ao quarto e/ou quinto fascículos mais caudais do músculo psoas. Provavelmente durante a introdução do cateter entre 15 a 18 centímetros, a cateterização privilegia a origem anatômica desses fascículos musculares como mostram as imagens (Figuras 2 e 3), similares às obtidas por Dupré ${ }^{27}$, Gaertner e col. ${ }^{28}$, Capdevila e col. ${ }^{29}$ embora grande massa muscular do psoas ter sido também documentada ${ }^{30}$. Neste caso, é de se supor que volumes anestésicos maiores rompam a tênue fascia muscular dos fascículos, resultando numa imagem global da espessura do músculo psoas, delimitando-o, parcial ou totalmente ${ }^{8}$.

As evidências tomográficas (Figuras 4 e 5), tridimensionais complementares, documentaram nos planos axiais transversos que a dispersão do contraste pela extremidade do cateter longo às zonas $\mathrm{A}, \mathrm{B}$ e C (Figura 1), coincidiram com as manifestações clínicas dos pacientes, embora o mesmo resultado tenha sido obtido quando o contraste se alojou alternativamente nas zonas radiológicas Ae C (Figuras 4 e 5). As deposições do contraste contíguo às estruturas ósseas, musculares e vasculares vizinhas ${ }^{18,19}$ são valiosas no estudo tomográfico pois, em função do trajeto anatômico do plexo lombossacro, permite-nos interpretar o resultado do bloqueio "3 em 1".

Com cateter longo (Contiplex ${ }^{\circledR}$ da B. Braun, 18G, $40 \mathrm{~cm}$ de comprimento) ou cateter peridural $18 \mathrm{G}$, os resultados foram um pouco diferentes. Por terem sido os cateteres introduzidos no sentido cranial entre 15 a $18 \mathrm{~cm}$ no espaço subfascial ilíaco, enrolamentos e/ou obras são acontecimentos que podem ocorrer, impedindo progressão intencional à região paravertebral lombar. No entanto, constatou-se no presente estudo que dois dos quinze cateteres longos $(13,2 \%)$ introduzidos sem guia, alojaram-se na zona C (paravertebral) (Figura 2), estando em conformidade não apenas com o formato opacificado paralombar do músculo psoas na altura de $\mathrm{L}_{5}-\mathrm{L}_{4}$ como demonstrado 15,27-29 mas também com a freqüência entre $11,76 \%{ }^{30} \mathrm{e}$ $22 \%{ }^{31}$ dos cateteres que aportaram nessa região. Cateteres introduzidos com guia metálico ${ }^{33}$ pela técnica de Seldinger ${ }^{34}$ determinam um posicionamento final mais programado e confiável que aleatório, traduzindo-se em resultados mais previsíveis de um bloqueio " 3 em 1 ". Contudo, avançar o cateter demasiadamente pode resultar num bloqueio peridural ${ }^{35}$, sendo a recíproca também verdadeira, como foi recentemente divulgado ${ }^{36}$.

Dos pacientes observados nesta pequena amostra, dois foram alvo de envolvimento das raízes lombares e do tronco lombossacro $\left(L_{4}-L_{5}\right)$. Representantes do plexo lombar e sacral originam o nervo ciático $\left(\mathrm{L}_{4}-\mathrm{S}_{3}\right)$, composto pelo nervo fibular comum $\left(L_{4}-S_{2}\right)$ e nervo tibial $\left(L_{4}-S_{3}\right)$ fisicamente independentes entre si porém envoltos por uma bainha comum ${ }^{37}$. Parece que as características físicas do cateter (Teflon ${ }^{\circledR}$ semirígido) favorecem sua progressão pelo compartimento subfascial ilíaco. Nesses pacientes, clichês radiográficos (Figura 1) e tomográficos complementares (Figuras 4 e 5) registraram a correlação radiológica entre os resultados clínicos e a anatomia pélvica correspondente.
A exemplo de prévias comunicações, constatou-se que, com injeção única de 30 a $40 \mathrm{ml}$ de solução anestésica, os resultados surpreendentes de outros estudos não foram observa$\operatorname{dos}^{38,39}$ pois, 1) a dispersão anestésica não ascende suficientemente; 2) o nervo femoral invariavelmente é acometido, já que a solução anestésica é injetada em seu trajeto, 3) o nervo fêmoro cutâneo lateral é atingido com uma incidência pouco menor em relação ao nervo femoral, pois a difusão da solução anestésica se difunde, preferencialmente mais para a face pélvica lateral que medial, e 4) o nervo obturador é eventualmente ou mesmo impossível de ser anestesiado, por se encontrar mais posterior em seu compartimento fascial estanque, de difícil acesso. Além disso, quando se observou alteração sensorial do dermátomo na face medial de toda a coxa, território inervado por ramos cutâneos do nervo femo$\mathrm{ral}^{12}$, a motricidade passiva (manutenção do joelho dobrado sobre a mesa sem pender lateralmente) ${ }^{8}$ e ativa dos músculos adutores (adução da coxa) ${ }^{8}$ encontravam-se preservadas. Os resultados obtidos na presente observação coincidiram com os recentemente divulgados sobre o envolvimento isolado do ramo anterior do nervo obturador 40,41

O trabalho de Winnie e col. ${ }^{1}$ trouxe um enriquecimento ao armamentário anestesiológico regional. A crescente preferência pela analgesia pós-operatória através do bloqueio "3 em 1" em cirurgias da coxa (enxerto de pele), fraturas/osteotomias do terço médio do fêmur e principalmente do joelho (ligamento cruzado anterior e prótese total de joelho), vem se tornando um dos procedimentos mais praticados no membro inferior. Contudo, o envolvimento anestésico do nervo obturador continua sendo controvertido, mais do ponto de vista anatômico do que do ponto de vista clínico-anestesiológico. Para se definir o comprometimento anestésico do nervo obturador (nervo misto) deve-se ter conduta e critérios clínicos bem definidos ${ }^{20}$. Infelizmente, na maioria dos trabalhos, prioriza-se apenas o acometimento sensitivo (dermátomo) desprezando a parte motora (miótomos) do nervo obturador, provavelmente porque os objetivos visam mais a analgesia pós-operatória com relaxamento reflexo do quadríceps femoral inervado pelo nervo femoral do que relaxamento muscular dos adutores da coxa inervados pelo nervo obturador. Um outro critério bastante aceitável sobre o envolvimento do nervo obturador no bloqueio "3 em 1" seria a correlação entre a clínica anestesiológica com os resultados radiológicos e tomográficos contrastados ${ }^{8}$. Embora a tomografia computadorizada nos proporcione um estudo tridimensional e as incidências radiográficas contrastadas, um estudo unidimensional sobre a dispersão do contraste, estas imagens projetadas sobre os diversos elementos anatômicos da região lombopélvica facilitam a interpretação sobre o desfecho clínico do bloqueio "3 em 1". Considerando os dados disponíveis na literatura em que o contraste situou-se na parte lateral da grande pelve em $37 \%$ dos casos ${ }^{29}$, observou-se bloqueios sensitivo e motor do nervo obturador, respectivamente em $27 \%$ e $22 \%$ dos casos 33,40 , superior aos $13,2 \%$ observados nesta pequena amostra. Face a isso, pode-se deduzir que: 
1) A dispersão da solução anestésica no espaço subfascial ilíaco, durante o bloqueio femoral "3 em 1", com injeção única ou com cateter, é aleatória e imprevisível ${ }^{41}$.

2) Sob injeção única o bloqueio que se obtém é, antes de tudo um bloqueio "1 em 1" ou exclusivamente femoral, em $100 \%$ dos casos, ou um bloqueio "2 em 1" ${ }^{42}$ sem a participação do nervo obturador, ou ainda, numa baixíssima incidência (1,3\%), um bloqueio "3 em 1" 43 .

3) Para a inserção de cateter longo no espaço subfascial ilíaco não há necessidade de auxílio de estimulador de nervo periférico ${ }^{21,44,45}$. Com o cateter longo posicionado medialmente (zona pélvica medial) ou na região paravertebral lombar (Zona radiológica $\mathrm{C}$ ), o resultado anestésico torna-se mais previsível podendo o bloqueio "3 em 1" assumir um caráter superdimensionado sob forma de um bloqueio "3,5 em 1", compreendendo os nervos cutâneo lateral da coxa, femoral, obturador e adicionalmente, um dos nervos, o ramo femoral do nervo genitofemoral ${ }^{5}$ ou o nervo fibular comum ${ }^{6,46}$.

4) Reportando Singelyn e col., um bloqueio anterior " 3 em 1 " excepcionalmente, identifica-se como um bloqueio peridural $^{35}$.

Adicionalmente, a partir da pequena amostra desse estudo pode-se concluir que:

1) Com injeção única sempre se obteve um bloqueio "2 em 1 " ou " 2,5 " (com envolvimento do ramo femoral do nervo genitofemoral) sem a participação do nervo obturador;

2) Com cateter curto (cateter venoso), um bloqueio "3 em 1" foi obtido em $6,6 \%$ dos casos (um paciente);

3) Com cateter longo introduzido através de cateter curto servindo como guia, obteve-se bloqueio " 3 em 1" em $20 \%$ dos casos (três pacientes), quando o primeiro aportou no espaço paravertebral lombossacro, ou, raramente um bloqueio "3,5 em 1" superdimensionado em $13,32 \%$ dos casos (dois pacientes), com envolvimento adicional do nervo fibular comum ou do nervo tibial (Figuras 4 e 5).

\section{AGRADECIMENTOS}

O autor agradece aos acadêmicos Martin Geier e Gustavo Andreazza Laporte pelo auxílio ao presente trabalho.

\section{Anterior "3-in-1" Blockade: Partial, Total or Overdimensioned Block? Correlation between Anatomy, Clinic and Radio Images}

\author{
Karl Otto Geier, M.D.
}

\section{INTRODUCTION}

Anterior 3-in-1 blockade, originally presented by Winnie et al. in $1973^{1}$, aimed at simultaneously anesthetizing with a single injection femoral, femoral lateral cutaneous of thigh and obturator nerves, components of the lumbar plexus, by accessing paravascular femoral space right below the inguinal ligament. Technique would involve the triggering of paresthesias with needle on the crural nerve, followed by 20 or more $\mathrm{mL}$ of anesthetic solution injection. It was recommended to press tissues immediately distal to the needle aiming at favoring anesthetic solution spread in the iliac subfascial space during injection. Initially results were successful ${ }^{1}$, with total 3 -in- 1 blockade. However, as the technique has become popular, it has been observed in the clinical practice that the involvement of the three nerves after single injection was not always true. Several publications have shown that the obturator nerve ${ }^{2}$ would fail more frequently than the lateral cutaneous of thigh ${ }^{3}$, as opposed to the femoral nerve ${ }^{4}$, characterizing a partial 3 -in-1 block or 2-in-1 block. However, very uncommon ipsilateral and overdimensioned 3 -in-1 blocks have been reported ${ }^{5,6}$. Oriented by superficial anatomic references suggested by Vloka et al. ${ }^{7}$, it has been observed in 45 consecutive 3 -in-1 blockades with single injection or catheters, the partial and ipsilateral involvement of the lumbar plexus. Clinical outcomes identifying total or overdimensioned 3-in-1 blockades face to the additional involvement of lumbosacral nerves were correlated to the anatomy and to a corresponding radio image study.

\section{METHODS}

After the Hospital Ethics Committee and patients' approval, forty five 3-in-1 blockades were induced through paravascular femoral approach in three groups of 15 patients, with glass or plastic syringes adequate for loss of resistance to air test, disposable sharp tip needles, short catheters with terminal hole and long catheters with three lateral holes:

Group 1 - fifteen 3-in1- blockades performed with single injection and disposable $35 \times 7$ or $40 \times 7$ needle coupled to a special syringe for loss of resistance to air test. The needle-syringe set was introduced in $35^{\circ}$ to $45^{\circ}$ angle with relation to skin until the iliac subfascial space, using $1.5 \%$ lidocaine with 1:300,000 epinephrine (30 to $40 \mathrm{~mL}$ ).

Group 2 - fifteen patients submitted to 3 -in-1 blockades with $18 \mathrm{G}$ short venous catheters, being six B. Braun's $45 \mathrm{~mm}$ length Introcan ${ }^{\circledR}$ and nine Nipro Medical Ltda's $50 \mathrm{~mm}$ length catheters, both with Quincke tip, introduced in acute angle similar to group 1. At iliac subfascial space identification, equivalent to the second loss of resistan$c e$, half anesthetic volume was injected, venous catheter 
needle was immobilized and catheter was cranially introduced throughout its extension, by clock and counterclock rotation movements along the needle's axis, followed by venous catheter needle removal. Anesthetic volume injected would help catheters introduction. It has been administered $0.25 \%$ bupivacaine with $1: 400,000$ epinephrine (30 to $40 \mathrm{~mL}$ ).

Group 3 - 3-in-1 blockades were performed with long 18G epidural catheters, being nine B. Braun's Perifix ${ }^{\circledR}$ and six Portex's Minipak ${ }^{\circledR}$. All catheters were cranially introduced 15 to $18 \mathrm{~cm}$ in the iliac subfascial space. Perifix ${ }^{\circledR}$ catheters through their guide catheter, without peripheral nerve stimulator help, and Minipak ${ }^{\circledR}$ catheters through the cannula of an $18 \mathrm{G}$ venous catheter, all previously introduced as described for group 2. An antibacterial filter was coupled to all catheters, anesthetic solution has been $0.25 \%$ bupivacaine with 1:400,000 epinephrine $(40 \mathrm{~mL})$.

Sensory and motor evaluation of 3-in-1 block was achieved by dermatomes (cutaneous innervation) or miotomes (muscle innervation) analgesia in burns and wounds, all with surgical indication, and sclerotomes (bone innervation) in thigh trauma patients with fractured of the femur medium third and knee. When skin, muscle and bone sensory investigation was impossible due to bandages or immobilization, pain relief itself has been the effective referential for 3-in-1 blockade. For elective surgeries under analgesia through catheters extending to the postoperative period, dermatomes sensitivity evaluation has followed the sequence: pain (hair follicles traction/needle pinprick), temperature (ice) and touch, followed by miotomes motility as already described ${ }^{8}$, provided they were compatible with the injury. All clinical investigations were compared to the homologous side.

Anatomic evaluation was coupled to radiological evaluation, the latter performed in blockades with short or long catheters (epidural and Contiplex ${ }^{\circledR}$ ), and resulted in clinical involvement of the classic three nerves and one additional nerve. Initially, 1 to $2 \mathrm{~mL}$ contrast would identify the catheter in the iliac subfascial space and then a higher volume of contrast (Omnipaque ${ }^{\circledR} 300 \mathrm{mg} / \mathrm{mL}$ ) diluted with local anesthetic would record spread in the possible three lumbopelvic radiological zones: zone A (medial pelvic), zone B (lateral pelvic) and zone $\mathrm{C}$ (paravertebral) ${ }^{5}$ (Figure 1 ). If radiological investigation overlapped the anatomic pathway of interested nerves, a CT-scan study was performed.

\section{Statistical Analysis}

Demographics data are shown in mean \pm SD. For categorical variables of three groups results are in relative frequencies, followed by chi-square test to determine significance of results, considering significant $p<0.05$.

\section{RESULTS}

Demographics data and procedures for the three groups are shown in tables I and II.

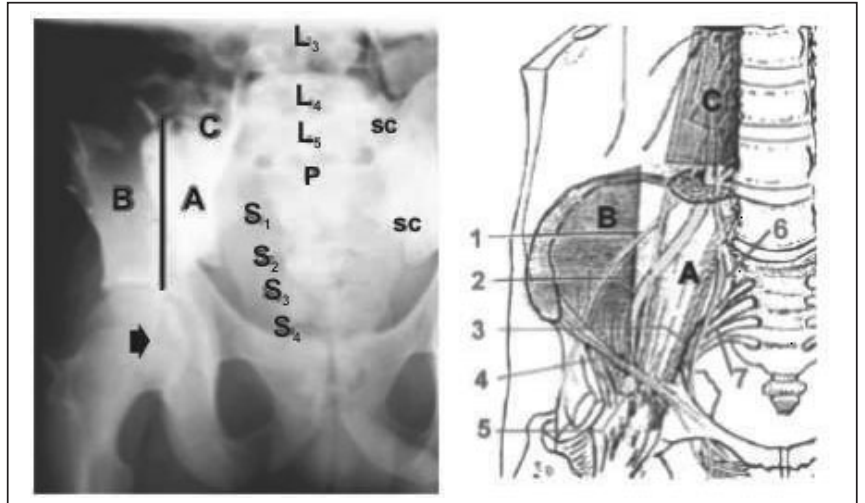

Figure 1 - Representation of Radiological Zones A, B, C where 3-in-1 Blockade Components Travel by Femoral Pathway Left: Contrast impregnation in anterior-posterior incidence may reveal individual or participative involvement of three classic nerves of 3-in-1 blockade. Arrow = catheter; P = Sacral promontory; $\mathrm{SC}=$ Sacral iliac joint; $\mathrm{S}_{1} ; \mathrm{S}_{2} ; \mathrm{S}_{3} ; \mathrm{S}_{4}=$ sacral foramens; $L_{3}, L_{4}, L_{5}=$ vertebral bodies. Right: 1 = lateral cutaneous nerve of thigh; 2 = femoral nerve; 3 = obturator nerve; 4 = genitofemoral nerve; $5=$ puncture site; $6=$ lumbosacral trunk; $7=$ sacral plexus

Table I - Demographics Data (Mean \pm SD)

\begin{tabular}{lccc}
\hline Groups & Age & Height & Weight \\
\hline G1 - Single Injection & $27.50 \pm 7.01$ & $169.33 \pm 8.19$ & $67.58 \pm 5.42$ \\
G2 - Short Catheter & $27.17 \pm 6.13$ & $166.58 \pm 6.01$ & $65.42 \pm 4.95$ \\
G3 - Long Catheter & $23.67 \pm 4.70$ & $171.92 \pm 9.88$ & $70.92 \pm 8.48$ \\
\hline
\end{tabular}

Table II - Procedures Performed in the Three Groups

\begin{tabular}{|c|c|c|c|}
\hline Indications & $\begin{array}{c}\text { G1=Single } \\
\text { Injection \#*。 }\end{array}$ & $\begin{array}{l}\text { G2=Short } \\
\text { Catheter }\end{array}$ & $\begin{array}{l}\text { G3=Long } \\
\text { Catheter }\end{array}$ \\
\hline
\end{tabular}

Skin donor area *

Change of dressings *o

Serial debridement * $。$

02

Femur medium third

fracture \#

Hip fracture * 。

Knee fracture *。

Internal saphenous nerve neuralgia •

Magnum saphenous vein extraction $^{\circ}$

Adductors of thigh

myalgia

Spider bite pain

01

$\mathrm{n}=15 \quad \mathrm{n}=15$

$\mathrm{n}=15$

All catheters were $18 \mathrm{G}$ with disposable $35 \times 7$ needle;

* (postoperative analgesia); ${ }^{\circ}$ (surgery/painful procedures);

\# (preoperative pain); • (chronic pain).

Femoral dermatome (mixed nerve) involvement was frequent, with femoral quadriceps muscle weakness, and almost as frequently there has been loss of lateral cutaneous of thigh dermatome (sensory nerve) sensitivity. In some cases, there has been loss of sensitivity on the medial face of almost 
Table III - Peripheral Nerves Involvement of Anterior 3-in1 Blockade ( $\mathrm{n}=45)$

\begin{tabular}{|c|c|c|c|c|c|c|}
\hline & \multicolumn{3}{|c|}{ 3-in-1 Blockade } & \multicolumn{3}{|c|}{ Additional Nerve to 3-in-1 Blockade } \\
\hline & $\begin{array}{l}\text { Femoral } \\
\text { Nerve (S/M) }\end{array}$ & $\begin{array}{l}\text { Lateral Cutaneous } \\
\text { Nerve of Thigh (S) }\end{array}$ & $\begin{array}{c}\text { Obturator Nerve } \\
(\mathrm{S} / \mathrm{M})\end{array}$ & $\begin{array}{l}\text { Genitofemoral } \\
\text { Nerve (S) }\end{array}$ & $\begin{array}{c}\text { Common Fibular } \\
\text { Nerve }(\mathrm{S} / \mathrm{M})\end{array}$ & $\begin{array}{l}\text { Tibial Nerve } \\
\text { (S/M) }\end{array}$ \\
\hline G1 - Single Injection $(n=15)$ & $15 \backslash 15(100 \%)$ & $13 \backslash 15(86.6 \%)$ & $0 \backslash 15$ & $4 \backslash 15(26.4 \%)$ & $0 \backslash 15$ & $0 \backslash 15$ \\
\hline G2 Short Catheter $(n=15)$ & $15 \backslash 15(100 \%)$ & $14 \backslash 15(93.2 \%)$ & $1 \backslash 15(6.6 \%)$ & $7 \backslash 15(46.6 \%)$ & $0 \backslash 15$ & $0 \backslash 15$ \\
\hline G3 Long Catheter $(n=15)$ & $15 \backslash 15(100 \%)$ & $13 \backslash 15(86.6 \%)$ & $2 \backslash 15(13.2 \%)$ & $7 \backslash 15(46.6 \%)$ & $2 \backslash 15(13.2 \%)$ & $\begin{array}{c}1 \backslash 15(6.6 \%) \\
\text { medial plantar }\end{array}$ \\
\hline$p$ value & - & $p<0.799$ & $p<0.146$ & $p<0.435$ & $p<0.123$ & $p<0.360$ \\
\hline
\end{tabular}

(S) Sensitivity; (M) Motility; Nerves Involvement among groups: NS (chi-square test)

the whole thigh where femoral and obturator dermatomes are overlapped with imprecise limits, however not always followed by adductor muscle paresis. Depending on lower limb morbidity, active (thigh bending - femoral nerve, and thigh adduction - obturator nerve) and passive (maintain bent knee with plantar region supported on bed, without pending the limb laterally - obturator nerve) motility were also evaluated as previously described ${ }^{8}$. Analgesia and anesthesia of additional dermatomes to the three classic 3-in-1 blockade nerves were observed in the territories of the femoral branch of genitofemoral nerve in 7 patients $(46.6 \%)$ and in the common fibular nerve territory in 2 patients $(13.2 \%)$, characterizing overdimensioned 3.5-in-1 block. One patient had additional partial tibial nerve involvement $(6.6 \%)$, practically becoming a 4-in-1 blockade (Table III).

Notwithstanding the 15 so-called 3 -in-1 blockades with single injection have not resulted in obturator nerve motor involvement, such blockades were considered 2 -in-1, so no radiological study was performed with the repetition of the same local anesthetic volume and mass. However, the 30 patients with short catheters (15) and long catheters (15) installed in the subfascial space of pelvic-lumbar muscles, who have clinically manifested loss of sensitivity in ectopic dermatome(s) to 3-in-1 blockade, were initially investigated by antero-posterior X-rays with 1 to $2 \mathrm{~mL}$ contrast to identify catheter positioning (Figure 2).

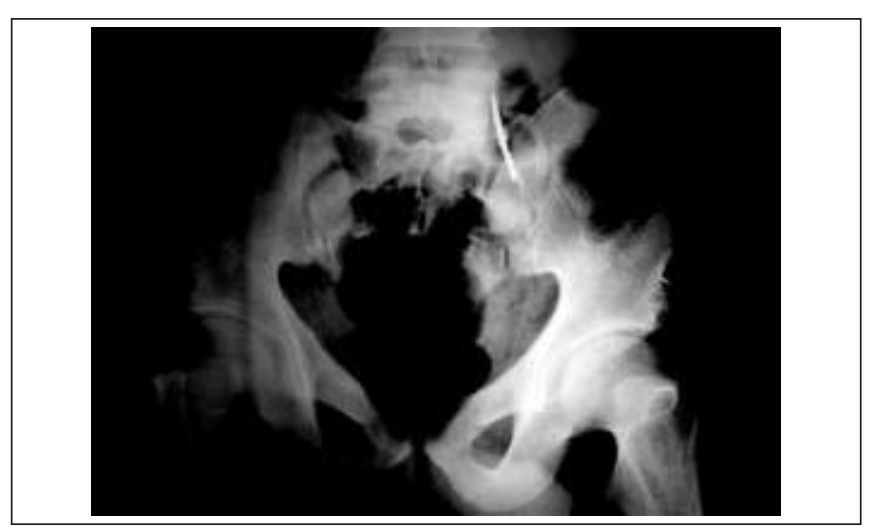

Figure 2 - One to Two $\mathrm{mL}$ Contrast by Contiplex ${ }^{\circledR}$ Catheter Reveal its Position in Left Radiological

Zones $A$ and $C$ in a patient with Painful involvement in the obturator nerve territory. Sacral-iliac joint is visible being considered a radiological differential for obturator nerve pathway
Among patients with short catheters, only one has developed total 3-in-1 blockade (true) with changes in sensitivity of one extra dermatome to the three classic 3 -in-1 blockade nerves. Contrasted X-ray images have identified pelvic radiological zones A (psoas muscle subfascial space) and B (iliac muscle subfascial space), however without medial ascending by zone A. Contrast has never reached zone $C$ (paravertebral region). On the other hand, for being long catheters aimed at a more cranial introduction between 15 and $18 \mathrm{~cm}$ in the iliac subfascial space, they have not shown clinical manifestations suggesting cranial ascension to radiological zone $\mathrm{C}$ with obturator nerve anesthesia. The trend to coiling and bending during introduction of these catheters does not allow their introduction to the lumbar paravertebral region, where the three nerves are confined, what explains the lack of obturator nerve blockade.

From 15 long catheter patients, two had catheter positioned at $L_{5}-L_{4}$ (Figure 3 ) in a frequency of $13.2 \%$. CT-scans of these patients have shown contrast in radiological zones $A, B, C$ (Figures 4 and 5 ) with the involvement of additional dermatomes to those of 3-in-1 blockade.

This was observed in a patient with traumatic avulsion of foot dorsum skin and submitted to grafting and in whom 3-in-1

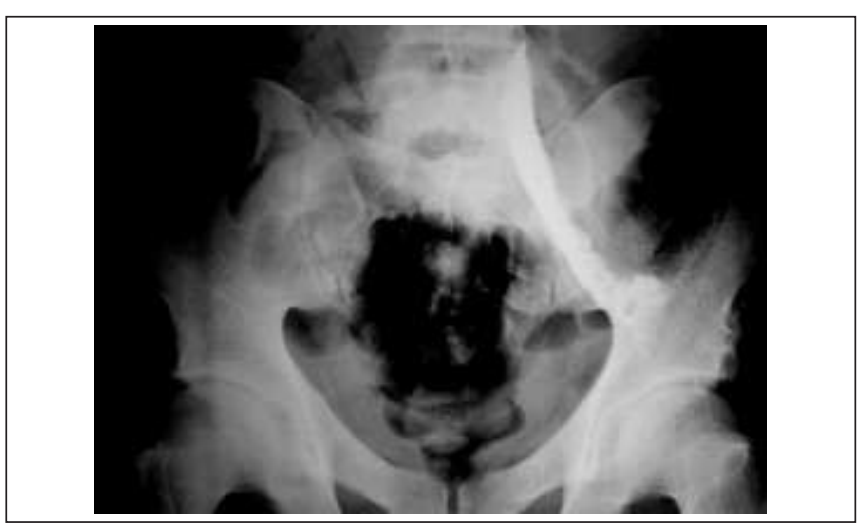

Figure 3 - Same Patient from figure 2 Identifying Local Anesthetic Mixed to Contrast with Spread throughout the $5^{\text {th }}$ Psoas Muscle Fascicle, to Radiological Zones

A and $\mathrm{C}$, corresponding to clinical findings of 3.5-in-1 blockade with involvement of lateral cutaneous of thigh and femoral nerves (both in radiological zone B), obturator nerve (radiological zone $A$ and $C$ ) and femoral branch of the genitofemoral nerve (transitional radiological zones $\mathrm{A}$ and $\mathrm{B}$ ) and lumbosacral trunk $\left(\mathrm{L}_{4}-\mathrm{L}_{5}\right)$ 


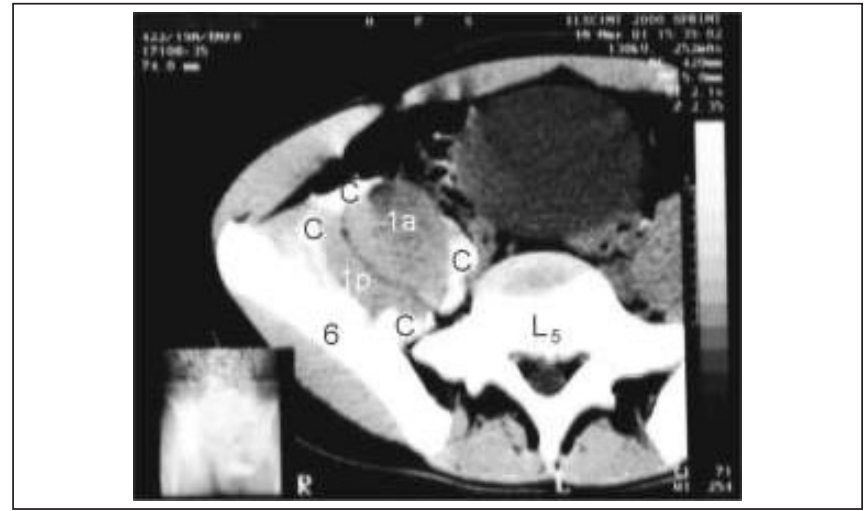

Figure 4 - TC-Scan Image in $\mathrm{L}_{5}$ of One of Two patients with Overdimensioned 3-on-1 Blockade Involving Lumbosacral Trunk and Resulting in Anesthesia of the common Fibular Nerve and Partial Anesthesia of the Tibial Nerve

Contrast $(\mathrm{C})$ involves and spread in the iliac muscle and both portions of the psoas muscle; $1 \mathrm{a}=$ larger or anterior portion of the psoas muscle; $1 \mathrm{~b}=$ smaller or posterior portion of the psoas muscle; 6 = iliac bone

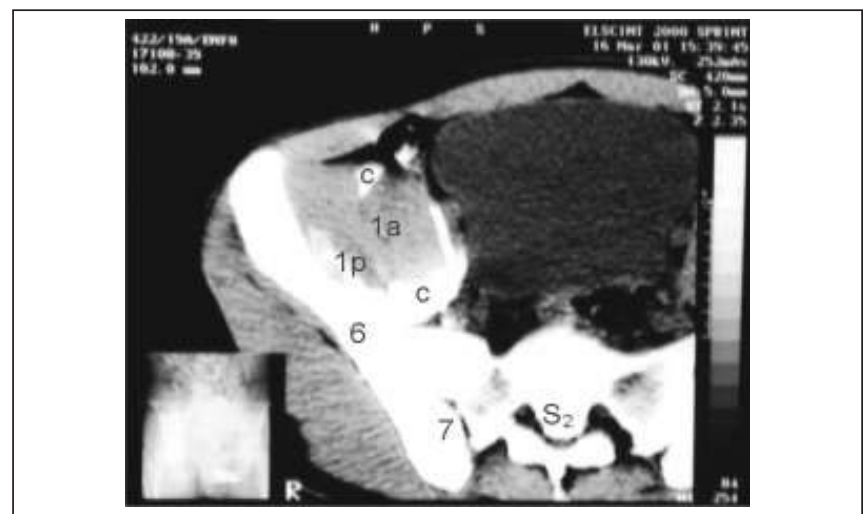

Figure 5 - TC-Scan Image in $\mathrm{S}_{2}$ of the Same Patient from figure 4 whose Contrast (C) has reached Lumbosacral trunk Resulting in Anesthesia of common Fibular and Tibial Nerve

$\mathrm{C}=$ contrast spreading in the iliac muscle and both portions of the psoas muscle at sacral iliac joint, partially visible (7); $1 \mathrm{a}=$ larger or anterior portion of the psoas muscle; $1 \mathrm{~b}=$ smaller or posterior portion of the psoas muscle; 6 iliac bone

blockade aimed at intermittent postoperative analgesia of the antero-lateral of thigh donor area. For our surprise, in addition to being effective for the donor area, patient has referred loss of tactile / painful sensitivity on the whole lateral face of leg and foot dorsum, corresponding to common fibular nerve dermatome $\left(L_{4}-L_{5}-S_{1}-S_{2}\right)$, followed by sensation of foot increased volume and temperature, characteristic of sympathetic block. This same patient was unable to cross the lower target limb over its homologous due to intense motor block of adductor muscles (obturator nerve), although mildly bending foot and toes (posterior tibial nerve) as opposed to the other foot. Loss of sensitivity in the plantar medial face was confirmed in this patient, corresponding to tibial nerve dermatome. CT images (Figures 4 and 5 ) have shown diffusion to sacral zones $\mathrm{C}$ and $\mathrm{A}\left(\mathrm{S}_{2}\right)$ (Figure 5 ).

\section{DISCUSSION}

Anatomically, lower limb innervation is made up of lumbar plexus $\left.\left[\left(T_{12}\right)-L_{1}-L_{5}\right)\right]$ and sacral plexus $\left(L_{4}-S_{3}\right)^{9,10}$. The union of these two plexuses is mediated by lumbosacral trunk $\left(L_{4}-L_{5}\right)$. Femoral $\left(L_{2}-L_{4}\right)$ and obturator $\left(L_{2}-L_{4}\right)$ dermatomes, the latter predominantly motor (through $L_{3}$ and $L_{4}$ roots), overlap in the internal face of thigh without precise limits ${ }^{11}$ or with more proximal distribution ${ }^{12}$ than distal, or even absent ${ }^{13}$. According to Bouaziz et al. ${ }^{14}$ obturator's dermatome is absent in almost $60 \%$ of cases. The issue gets more important in one Gray's book edition ${ }^{9}$ where the author does not mention obturator dermatome. Confusion between obturator and femoral dermatome still persists ${ }^{15}$. Femoral and lateral cutaneous of thigh nerves are located more laterally between iliac and psoas muscles and the iliac fascia involving them ${ }^{16}$. While distal iliac fascia delimits paravascular femoral space in the inguinal region, obturator nerve by going from its origin to the lower limb, is more posterior, outside this space and medially to great psoas muscle ${ }^{17}$, making difficult its approach by single injection, differently from when anesthetic solution is released by the tip of a catheter positioned in the radiological zone $\mathrm{A}$, or even posteriorly where the anesthetic is deposited directly in radiological zone C. The obturator nerve follows its caudal pathway not always following femoral and lateral cutaneous of thigh nerves. At $L_{4}-L_{5}$, while the former runs, at variable frequencies, outside or peripherally between the two parts of great psoas muscle, both covered by a thin fascia ${ }^{17,18}$, the two latter have a more constant relationship between them within the muscular mass ${ }^{19}$, separating at the sacral promontory $\left(L_{5}-S_{1}\right)$. The obturator nerve goes to the pelvis minor and the other two to the pelvis major. At the foramen receiving its name, the obturator nerve is divided in two branches: anterior or superficial branch and posterior or deep branch. Anterior or superficial branch is directed to hip joint and medial cutaneous surface of the leg, which in anastomosing with the internal saphenous nerve and multiple medial cutaneous branches of the femoral nerve, make up the subsartorial plexus, whose sensory cutaneous projection generate confusions on sensory tests of their corresponding dermatomes. In the medial third of the thigh, the anterior or superficial branch, predominantly sensory, goes toward superficial tissues with poor motor participation on long adductor, gracillis and short adductor muscles ${ }^{11}$. Deep or posterior branch, predominantly motor, goes toward external obturator, adductor magum and short adductor muscles anastomosing with the superficial branch and projecting to the posterior face of the popliteal region and sinovial membrane of the knee. Most 3-in-1 block clinical observations only check sensory involvement and not motor involvement of the obturator nerve leading to misinterpretations about its extension ${ }^{20}$. Objective and well-defined criteria ${ }^{21}$ should then orient clinical research of all and any anesthetic block, checking sensory and motor abolishment, respectively, of superficial (cutaneous) and deep (muscles, joints, viscera and veins) tissues. 
Technical evidences of 3-in-1 blockade results confirm that, for the identification of the paravascular femoral space it is not always necessary to use peripheral nerve stimulator ${ }^{21}$ or paresthesias ${ }^{1}$ through needle contact with the nerve. Loss of resistance is an adequate method for identification of virtual spaces ${ }^{21-23}$ between tissues of different densities in several peripheral blocks ${ }^{24}$.

Different contrasted radiological imaging modalities for regional anesthesia are becoming important, intensively impacting the learning of regional anesthetic techniques ${ }^{16}$ for diagnosing and treating painful diseases ${ }^{25}$. In spite of unidimensional images, contrasted antero-posterior radiological evidences of the three radiographic zones ${ }^{5}$ should be interpreted based on contrast spread in the subfascial compartment of iliac and greater psoas muscles, through which nervous components of 3 -in- 1 blockade travel.

Obturator nerve distally crosses zone A (medial pelvic region), femoral nerve travels distally between zone $A$ and internal zone $B$ limit (lateral pelvic region) and lateral cutaneous of thigh nerve goes distally to zone A. Zone C (lumbar paravertebral region) corresponds to the proximal half of the psoas muscle ${ }^{5}$ (Figure 1), where with a single posterior injection is more feasible to anesthetize all three nerves involved in 3-in-1 blockade ${ }^{26}$, as compared to the anterior approach. In a 3-in-1 blockade with long catheters, contrasted images are in general long, spindle-like and well delimited, almost always ending laterally to vertebral bodies $L_{3}-L_{4}$ at the level of intervertebral discs, corresponding to the fourth or fifth more caudal fascicles of the psoas muscle. It is possible that during catheter introduction 15 to $18 \mathrm{~cm}$, catheterization privileges the anatomic origin of these muscle fascicles as shown in figures 2 and 3 , and similar to images obtained by Dupré ${ }^{27}$, Gaertner et al. ${ }^{28}$ and Capdevila et al. ${ }^{29}$ although the large psoas muscle mass has been also documented ${ }^{30}$. In this case, it is to be assumed that larger anesthetic volumes would break the thin fascicle muscle fascia resulting in a global image the thickness of the psoas muscle, partially or totally delimiting it ${ }^{8}$.

Tridimensional CT-scan evidences (Figures 4 and 5) have documented in transverse axial planes that contrast spread by long catheter tip to zones A, B and C (Figure 1), have coincided with patients' clinical manifestations, although the same result has been obtained when contrast was alternatively lodged in radiological zones $A$ and $C$ (Figures 4 and 5). Contrast deposition close to neighbor bone, muscle and vascular structures ${ }^{18,19}$ is valuable in the tomographic study because as a function of the anatomic pathway of lumbosacral plexus, it allows us to interpret the results of 3-in-1 blockades. With long catheter (B.Braun's Contiplex ${ }^{\circledR}, 18 \mathrm{G}, 40 \mathrm{~cm}$ length) or $18 \mathrm{G}$ epidural catheter, results were a little different. Because catheters are cranially introduced 15 to $18 \mathrm{~cm}$ in the iliac subfascial space, coiling and/or bending may occur, preventing the intentional progression to the lumbar paravertebral region. Our study, however, has observed that two out of 15 long catheters (13.2\%) introduced without guide, were lodged in zone $\mathrm{C}$ (paravertebral) (Figure 2) being in conformity not only with the paralumbar opaque format of psoas muscles at $L_{5}-L_{4}$ as shown ${ }^{15,27-29}$ but also with frequency between $11.76 \%{ }^{31}$ and $22 \%{ }^{32}$ of catheters reaching this region. Catheters introduced with metallic guides ${ }^{33}$ by Seldinger's ${ }^{34}$ technique have determined a final positioning more programmed and reliable than randomized, translating into more predictable 3-in-1 blockade results. However, advancing the catheter too much may result in epidural block ${ }^{35}$, being the opposite also true, as it has been recently published 36 .

From patients observed in this small sample, two had lumbar roots and lumbosacral trunk $\left(\mathrm{L}_{4}-\mathrm{L}_{5}\right)$ involvement. Lumbar and sacral plexuses representatives originate the sciatic nerve $\left(\mathrm{L}_{4}-\mathrm{S}_{3}\right)$ made up of common fibular nerve $\left(\mathrm{L}_{4}-\mathrm{S}_{2}\right)$ and tibial nerve $\left(L_{4}-S_{3}\right)$, physically separated but involved by a common sheath ${ }^{37}$. It seems that physical catheter characteristics (Teflon ${ }^{\circledR}$ semi-rigid) favor its progression through the iliac subfascial compartment. In these patients, additional radiographic (Figure 1) and tomographic (Figures 4 and 5) images have recorded the radiological correlation between clinical results and corresponding pelvic anatomy.

Similar to other publications, it has been observed that with single 30 to $40 \mathrm{~mL}$ anesthetic solution injection, surprising results of other studies have not been observed ${ }^{38,39}$ because 1) anesthetic spread does not sufficiently ascend; 2) femoral nerve is always involved because anesthetic solution is injected in its pathway; 3 ) lateral cutaneous femoral nerve is reached in a slightly lower incidence as compared to the femoral nerve because anesthetic spread is preferably diffused to the lateral pelvic face as compared to the medial face, and 4) obturator nerve is difficult or even impossible to be anesthetized because it is more posteriorly located in its difficult to access sealed fascial component. In addition, when sensory changes were observed in the dermatome of the medial face of the whole thigh, which is a territory innervated by femoral nerve cutaneous branches ${ }^{12}$, passive motility (maintaining the knee bent on the table without pending laterally $)^{8}$ and active motility of adductor muscles (thigh adduction) ${ }^{8}$ were preserved. Results of our study have coincided with recently published data on the isolated involvement of anterior obturator nerve branch ${ }^{40,41}$. Winnie et al. ${ }^{1}$ study has enriched regional anesthetic armamentarium. The increasing preference for postoperative analgesia via 3-in-1 blockade in thigh surgeries (skin grafting), fractures/osteotomies of femoral medium third and especially of the knee (anterior cruciate ligament and total knee replacement), is becoming one of the most performed procedures for lower limbs. However, the anesthetic involvement of the obturator nerve is still controversial, more from the anatomic than from the clinical-anesthetic point of view. To define the anesthetic involvement of the obturator nerve (mixed nerve) it is necessary to adopt well-defined approaches and clinical criteria ${ }^{20}$. Unfortunately, most studies prioritize only the sensory involvement (dermatome) overlooking the motor part (miotomes) of the obturator nerve, probably because the objectives aim at postoperative analgesia with reflex relaxation of femoral quadriceps innervated by the femoral nerve, and not muscle relaxation of adductor muscles of thigh innervated by the obturator nerve. Another acceptable criteria 
about obturator nerve involvement in 3-in-1 blockades would be the correlation between anesthetic clinic and contrasted radiological and tomographic results ${ }^{8}$. Although CT-scan provides tridimensional images and contrasted radiological provides a unidimensional study about contrast spread, these images projected on different anatomic elements of the lumbopelvic region help the interpretation of 3-in-1 blockade clinical outcomes.

Considering data available in the literature, in which contrast was located in the lateral side of the pelvis major in $37 \%$ of cases ${ }^{29}$, obturator nerve sensory and motor blocks have been observed in $27 \%$ and $22 \%$ of cases, respectively ${ }^{33,40}$, higher than $13.2 \%$ observed in our small sample. Face to this, one may assume that:

1) Anesthetic solution spread to the iliac subfascial space during 3-in-1 femoral blockade with single injection or catheter is always random and unpredictable ${ }^{41}$.

2) With single injection, blockade obtained is before all 1-in-1 blockade, or exclusively femoral, in $100 \%$ of cases, or 2-in-1 blockade ${ }^{42}$ without obturator nerve participation, or even, in a very low incidence (1.3\%), 3-in-1 blockade ${ }^{43}$.

3 ) There is no need for peripheral nerve stimulator to insert the long catheter in the iliac subfascial space ${ }^{21,44,45}$. With the long catheter medially positioned (medial pelvic zone) or in the lumbar paravertebral region (radiological zone $\mathrm{C}$ ) anesthetic results are more predictable and 3-in-1 blockade may be overdimensioned as 3.5-in-1 blockade with the involvement of lateral cutaneous of thigh, femoral and obturator nerves and, additionally the femoral branch of the genitofemoral nerve ${ }^{5}$ or the common fibular nerve ${ }^{6,46}$.

4) According to Singelyn et al., an anterior 3-in-1 blockade is exceptionally identified as epidural block ${ }^{35}$.

Additionally, as from this small sample, it is possible to conclude that:

1) Single injection has always resulted in 2 -in-1 or 2.5 -in-1 blockade (with the involvement of the femoral branch of the genitofemoral nerve) without obturator nerve participation;

2) Short catheter (venous catheter) has resulted in 3-in-1 blockade in $6.6 \%$ of cases (one patient);

3) Long catheter introduced through the short catheter acting as a guide has resulted in 3-in-1 blockade in $20 \%$ of cases ( 3 patients), when the former has reached the paravertebral space, or more uncommonly, overdimensioned 3.5 -in/1 blockade in $13.32 \%$ of cases (2 patients) with additional involvement of the common fibular nerve or of the tibial nerve (Figures 4 and 5).

\section{ACKNOWLEDGEMENTS}

The author acknowledges students Martin Geier and Gustavo Andreazza Laporte for their help in this study.

\section{REFERÊNCIAS - REFERENCES}

01. Winnie AP, Ramamurthy S, Durrani Z - The inguinal paravascular technic of lumbar plexus anesthesia: the "3-in-1 block". Anesth Analg, 1973;52:989-996.

02. Lang SA, Yip RW, Chang PC et al - The femoral 3-in-1 block revisited. J Clin Anesth, 1993;5:292-296.

03. Madej TH, Ellis FR, Halsall PJ - Evaluation of "3 in 1" lumbar plexus block in patients having muscle biopsy. $\mathrm{Br} \mathrm{J}$ Anaesth, 1989;62:515-517.

04. Ritter JW - Femoral nerve "sheath" for inguinal paravascular lumbar plexus block is not found in human cadavers. J Clin Anesth, 1995;7:470-473.

05. Capdevila X, Biboulet $P$, Bouregba $M$ et al - Comparison of the three-in-one and fascia iliac compartment blocks in adults: clinical and radiographic analysis. Anesth Analg, 1998;86: 1039-1044.

06. Mansour NY - 3-in-1 or 4-in-1? Reg Anesth, 1992;17:242-243.

07. Vloka JD, Hadzic A, Drobnik L et al - Anatomical landmarks for femoral nerve block: a comparison of four needle insertion sites. Anesth Analg, 1999;89:1467-1470.

08. Geier KO - Critérios de avaliação do bloqueio anestésico 3 em 1: é comum o envolvimento do nervo obturador? Rev Bras Anestesiol, 2003;53:501-511.

09. Gray H - Anatomia. $29^{a}$ Ed, Rio de Janeiro. Guanabara Koogan, 1977;806-819.

10. Netter FH - Atlas of Human Anatomy. New Jersey. Novartis. $19^{\text {th }}$ Ed, 1997;468-469.

11. Spalteholz W - Atlas de Anatomia Humana. $3^{a}$ Ed, Barcelona, Editorial Labor, 1967;898.

12. Gray S - Anatomy. $39^{\text {th }}$ Ed, London. Churchill Livingstone, 1989;1140-1143

13. Bridenbaugh PO - The Lower Extremity: Somatic Blockade, em: Cousins MJ, Bridenbaugh PO - Neural Blockade. $2^{\text {nd }}$ Ed, Philadelphia. JB Lippincott, 1988;417-441.

14. Bouaziz H, Vial F, Jochum D et al - An evaluation of the cutaneous distribution after obturator nerve block. Anesth Analg, 2002; $94: 445-449$.

15. Geier KO - Bloqueio 3 em 1 com bupivacaína a 0,25\% para analgesia pós-operatória em cirurgias ortopédicas. Rev Bras Anestesiol, 2001;51:176-177.

16. McQuillan PM - Obturator Nerve, em: Hahn MB, McQuillan PM, Sheplock GJ - Regional Anesthesia. St. Louis. Mosby-Year Book, 1996;147-149.

17. Netter FH - Atlas of Human Anatomy. Novartis. New Jersey. $19^{\text {th }}$ Ed, 1997;244,250,345.

18. Farny J, Drolet P, Girard M - Anatomy of the posterior approach to the lumbar plexus block. Can J Anaesth, 1994;41:480-485.

19. Dietemann JL, Sick H, Wolfram-Gabel R et al - Anatomy and computed tomography of the normal lumbosacral plexus. Neuroradiology, 1987;29:58-68.

20. Imbeloni LE - Bloqueio 3 em 1 com bupivacaina a 0,25\% para analgesia pós-operatória em cirurgias ortopédicas. Rev Bras Anestesiol, 2001;51:177-178.

21. Geier KO - Is a Peripheral Nerve Stimulator (PNS) Really Necessary to Block the Femoral Nerve? The International Monitor. Medicon International $19^{\text {th }}$ Annual ESRA Congress. Rome, 2000;12:91.

22. Khoo ST, Brown TC - Femoral nerve block - the anatomical basis for a single injection technique. Anaesth Intensive Care, 1983;11:40-42.

23. Edwards ND, Wright EM - Continuous low-dose 3-in-1 nerve blockade for postoperative pain relief after total knee replacement. Anesth Analg, 1992;75:265-267. 
24. Geier KO - Analgesia regional prolongada com catéteres periféricos. Relato de casos. Rev Bras Anestesiol, 2002;52: 62-73.

25. Rathmell JP - Imaging in regional anesthesia and pain Medicine: we have much to learn. Reg Anesth Pain Med, 2002;27: 240-241.

26. Parkinson SK, Mueller JB, Little WL - Extent of blockade with various approaches to the lumbar plexus. Anesth Analg, 1989;68:243-248

27. Dupré LJ - Bloc "3 en 1" or bloc femoral. Que faut-il faire et comment le faire ? Ann Fr Anesth Réanim, 1996;15:1099-1106.

28. Gaertner E, Petit S, Cuby C et al - Parasacral catheter placement for lower limb surgery. (ASRA $26^{\text {th }}$ Annual Meeting) Reg Anesth Pain Med, 2001;26:70.

29. Capdevila X, Biboulet $P$, Morau D et al - Continuous three-in-one block for the postoperative pain after lower limb orthopedic surgery: where do the catheters go? Anesth Analg, 2002;94: 1001-1006.

30. Anker-Moller E, Spangsberg N, Dahl JB et al - Continuous blockade of the lumbar plexus after knee surgery: a comparison of the plasma concentrations and analgesic effect of bupivacaine $0.250 \%$ and $0.125 \%$. Acta Anaesthesiol Scand, 1990;34: 468-472.

31. Moreau D, Barthelet Y, Ryckwaert Y et al - Continuous "3-in-1" or fascia iliaca compartment blocks: is there a difference in the obtained catheter migration and sensory block? (ASRA $26^{\text {th }}$ Annual Meeting). Reg Anesth Pain Med, 2001;26:52.

32. Capdevila $X$, Biboulet $P$, Barthelet $Y$ et al - Continuous lumbar plexus block: where does the catheter go? Br J Anaesth, 1998; 80:(Suppl1):A387.

33. Singelyn FJ, Gouverneur JMA - Extended "three-in-one" block after total knee arthroplasty: continuous versus patient-controlled techniques. Anesth Analg, 2000;91:176-180.

34. Seldinger SI - Catheter replacement of the needle in percutaneous arteriography. Acta Radiol, 1952;28:368-376.

35. Singelyn FJ, Contreras V, Gouverneur JM - Epidural anesthesia complicating continuous 3-in-1 lumbar plexus blockade. Anesthesiology, 1995;83:217-220

36. Sudbrack G, Geier KO - Cateter peridural deslocado: uma causa de falha de analgesia. Rev Bras Anestesiol, 2002;52:55-61.

37. Vloka JD, Hadzic A, April E et al - The division of the sciatic nerve in the popliteal fossa: anatomical implications for popliteal nerve blockade. Anesth Analg, 2001;92:215-217.

38. Marhofer P, Oismüller C, Faryniak B et al - Three-in-one blocks with ropivacaine: evaluation of sensory onset time and quality of sensory block. Anesth Analg, 2000;90:125-128.

39. Imbelloni LE, Gouveia MA - Analgesia pós-operatória com bloqueio do plexo lombar: comparação entre as técnicas com cateter e com estimulação do nervo femoral. Rev Bras Anestesiol, 2001;51:28-36.

40. Marhofer P, Nasel C, Kapral C - Magnetic resonance image of local anesthetic during three-in-one blocks. Anesthesiology, 1999;91:3:A899.

41. Cauhèpe $C$. Olivier $M$, Colombani R et al - Le Bloc "trois-en-un": mythe ou réalité? Ann Fr Anesth Réanim, 1989;8:376-378.

42. Parkinson SK, Mueller JB, Little WL et al - Lumbar plexus blocks and lumbar plexus nerve blocks. Anesth Analg, 1989;69:854.

43. Spillane WF - 3-in-1 blocks and continuous 3-in-1 blocks. Reg Anesth, 1992;17:175-176.
44. Geier KO - Bloqueio contínuo do plexo lombar via compartimento ilíaco, combinado com bloqueio contínuo do nervo femoral em trauma grave de membro inferior. Relato de caso. Rev Bras Anestesiol, 2001;51:53-58.

45. Geier KO - Identificação tomográfica da bainha epineural dos nervos poplíteos durante anestesia regional intermitente do pé. Relato de caso. Rev Bras Anestesiol, 2002;52:581-587.

46. Winnie AP - The 3-in-1 block: is it really 4 -in-1 or $2: 1$ ? Reg Anesth, 1992;17:176-179.

\section{RESUMEN}

Geier KO - Bloqueo "3 en 1" por Vía Anterior: Bloqueo Parcial, Completo o Superdimensionado? Correlación entre Anatomía, Clínica y Radio Imágenes

JUSTIFICATIVA Y OBJETIVOS: El clásico bloqueo "3 en 1 " por vía anterior ha suscitado divergencias cuanto al envolvimiento anestésico de sus tres participantes, el nervio femoral, el nervio cutáneo lateral del muslo y el nervio obturador. El objetivo de este estudio es verificar el resultado del bloqueo "3 en 1 " por vía anterior, a través de las técnicas: inyección única (G1), catéteres cortos (G2) y catéteres largos (G3). Los bloqueos "3 en 1", clínicamente identificados como completos o superdimensionados fueron, adicionalmente, investigados por medio de radio imagen.

MÉTODO: La identificación del espacio subfascial ilíaco en los bloqueos "3 en 1" con inyección única o con catéteres fue hecha por la pérdida de resistencia al aire. En varios eventos dolorosos, el volumen anestésico administrado varió entre $30 y$ $40 \mathrm{ml}$ y la introducción cranial de los catéteres fue hasta $18 \mathrm{~cm}$ en el espacio subfascial ilíaco. Cuando la pesquisa clínica apuntaba envolvimiento del nervio obturador o de otro nervio adicional al bloqueo "3 en 1", se complementava la investigación con estudio radiográfico y tomodensiométrico con el propósito de establecer correspondencia con la anatomía pélvica.

RESULTADOS: El envolvimiento de los nervios femoro cutáneo lateral y obturador no fueron constantes, al contrario del nervio femoral. En el estudio, ningún bloqueo "3 en 1" completo con inyección única (G1) se manifestó, y si un bloqueo "2 en 1", con la participación eventual del nervio genitofemoral (bloqueo "2,5 en 1"), uno de los ramos del nervio femoral. Todavía, cuando fueron utilizados catéteres cortos (G2), se obteve bloqueo "3 en 1 " en apenas un paciente, al paso que con catéteres largos (G3) introducidos en el sentido cefálico até $18 \mathrm{~cm}$ en el espacio subfascial ilíaco, tres bloqueos "3 en 1" superdimensionados fueron registrados, por el envolvimiento adicional de los nervios fibular común en dos pacientes y el nervio tibial en un paciente.

CONCLUSIONES: A pesar de la pequeña muestra, con inyección única (G1), siempre se obtuvo un bloqueo "2 en 1 "ó "2,5 en 1", sin la participación del nervio obturador. Con catéter corto (G2), el bloqueo "3 en 1 " fue clasificado como completo en $6,6 \%$ de los casos (un paciente). Mas, con catéter largo (G3), el resultado tiende a ser más previsible en relación a los otros grupos, especialmente cuando el catéter alcanza el espacio paravertebral lombosacral, resultando en un bloqueo "3 en 1" completo en $20 \%$ de los casos (tres pacientes) o, raramente, en un bloqueo "3 en 1 " superdimensionado en 13,2\% de los casos (dos pacientes). 